\title{
Automated Location Invariant Animal Detection In Camera Trap Images Using Publicly Available Data Sources
}

\author{
Andrew Shepley ${ }^{1}$, Greg Falzon ${ }^{2}$, Paul D. Meek $^{3}$, and Paul Kwan ${ }^{4}$ \\ ${ }^{1}$ University of New England School of Science and Technology \\ ${ }^{2}$ University of New England \\ ${ }^{3} \mathrm{NSW}$ Dept Primary Industries \\ ${ }^{4}$ Melbourne Institute of Technology
}

October 21, 2020

\begin{abstract}
1. A time-consuming challenge faced by ecologists is the extraction of meaningful data from camera trap images to inform ecological management. Automated object detection solutions are increasingly, however, most are not sufficiently robust to be deployed on a large scale due to lack of location invariance across sites. This prevents optimal use of ecological data and results in significant resource expenditure to annotate and retrain object detectors. 2. In this study, we aimed to (a) assess the value of publicly available image datasets including FlickR and iNaturalist (FiN) when training deep learning models for camera trap object detection (b) develop a for training location invariant object detection models and (c) explore the use of small subsets of camera trap images for optimization training. 3. We collected and annotated 3 datasets of images of striped hyena, rhinoceros and pig, from FiN, and used transfer learning to train 3 object detection models in the task of animal detection. We compared the performance of these models to that of 3 models trained on the Wildlife Conservation Society and Camera CATalogue datasets, when tested on out of sample Snapshot Serengeti datasets. Furthermore, optimized the FiN models via infusion of small subsets of camera trap images to increase robustness for challenging detection cases. 4. In all experiments, the mean Average Precision (mAP) of the FiN models was significantly higher (82.33-88.59\%) than that achieved by the models trained only on camera trap datasets (38.5-66.74\%). The infusion of camera trap images into FiN training further improved mAP, with increases ranging from 1.78-32.08\%. 5. Ecology researchers can use FiN images for training robust, location invariant, out-of-the-box, deep learning object detection solutions for camera trap image processing. This would allow AI technologies to be deployed on a large scale in ecological applications. Datasets and code related to this study are open source and available at: https://github.com/ashep29/infusion
\end{abstract}

\section{Hosted file}

Manuscript.pdf available at https://authorea.com/users/369040/articles/487977-automatedlocation-invariant-animal-detection-in-camera-trap-images-using-publicly-available-datasources 